\title{
AVALIAÇÃO DA DENSIDADE E DO PH DE GÉIS FLUORETADOS DISPONÍVEIS NO MERCADO NACIONAL
}

\author{
EVALUATION OF DENSITY AND PH OF FLUORIDE GELS AVAILABLE IN \\ NATIONAL MARKET
}

\begin{tabular}{|c|c|}
\hline VinícIUs PEDRAZZI & $\begin{array}{l}\text { Faculdade de Odontologia de Ribeirão Preto - USP. } \\
\text { pedrazzi@forp.usp.br }\end{array}$ \\
\hline Murillo Sucena Pita. & $\begin{array}{l}\text { Faculdade de Odontologia de Ribeirão Preto - USP. } \\
\text { murillosucenapita@usp.br }\end{array}$ \\
\hline Cássio Do Nascimento & $\begin{array}{l}\text { Faculdade de Odontologia de Ribeirão Preto - USP. } \\
\text { cassionasc@forp.usp.br }\end{array}$ \\
\hline Flávio Henrique Carriço Nogueira Fernandes & $\begin{array}{l}\text { Faculdade de Odontologia de Ribeirão Preto - USP. } \\
\text { flaviocarrico@forp.usp.br }\end{array}$ \\
\hline Jerônimo Manço de Oliveira Neto & $\begin{array}{l}\text { Faculdade de Odontologia de Ribeirão Preto -USP. } \\
\text { jeronimoneto@usp.br }\end{array}$ \\
\hline Paulo Linares Calefi & $\begin{array}{l}\text { Faculdade de Odontologia de Ribeirão Preto - USP. } \\
\text { pcalefi@hotmail.com }\end{array}$ \\
\hline
\end{tabular}

\section{RESUMO}

Atualmente, o emprego de fluoretos, seja na forma de administração sistêmica ou na forma de aplicação tópica, é ato pragmático e quase que uma unanimidade em termos de Odontologia Preventiva. Dentre as características dos géis fluoretados para aplicação tópica, a densidade e o $\mathrm{pH}$ destes produtos possuem importância muito significativa na segurança e eficácia deste procedimento preventivo. $\mathrm{O}$ objetivo do presente estudo foi analisar a densidade e o $\mathrm{pH}$ de géis fluoretados disponíveis no mercado nacional, de diferentes marcas comerciais e em duas concentrações distintas. Para a verificação das propriedades dos géis fluoretados presentes no mercado brasileiro, foram adquiridos 10 produtos de cinco diferentes fabricantes, separados em géis de fluoreto de sódio a $2 \%$, com caráter neutro, e géis de flúor fosfato acidulado (FFA) a 1,23\%, dados estes declarados pelos fabricantes e impressos nos rótulos das embalagens. Os valores de $\mathrm{pH}$ para os géis de fluoreto de sódio (2\%) estão rigorosamente dentro dos padrões estabelecidos para esses produtos. Já os géis de FFA (1,23\%) Odahcam ${ }^{\mathrm{O}}$ e Inodon $^{\mathrm{O}}$ apresentaram grandes diferenças de $\mathrm{pH}$, respectivamente $(4,96)$ e $(4,65)$, ou seja, acima de $30 \%$ entre o $\mathrm{pH}$ esperado e o encontrado. As densidades dos géis avaliados apontam para produtos com valores adequados ao fim a que se destinam (aplicação tópica de flúor supervisionada por cirurgião-dentista), entretanto os produtos Odahcam $^{\mathrm{O}}$ e Inodon ${ }^{\mathrm{O}}$, por apresentaram grandes diferenças de $\mathrm{pH}$, colocam-se fora do padrão mínimo de eficácia desejado.

Palavras-chave: CONCENTRAÇÃO DE ÍONS DE HIDROGÊNIO; FLUORETO DE FOSFATO ACIDULADO; FLUORETO DE SÓDIO.

\section{Abstract}

Currently, the use of fluoride either as systemic or topical is pragmatic and almost unanimity in terms of Preventive Dentistry. Among the characteristics of fluoride gels for topical application, the density and $\mathrm{pH}$ of these products have very significant importance on the safety and efficacy of this preventive procedure. The aim of this study was evaluate the density and $p H$ of fluoride gels available in the national market of different brands and two different concentrations. To check the properties of fluoride gels in the Brazilian market were purchased 10 products from 5 different manufacturers, separated in gels of sodium fluoride to $2 \%$, with its neutral and acidulated phosphate fluoride gel $(A P F)$ to $1.23 \%$, these data declared by manufacturers and printed on the packaging labels. The $\mathrm{pH}$ values for the gels of sodium fluoride (2\%) are strictly within the established standards for these products. Already gels APF (1,23\%) Odahcam ${ }^{\circledR}$ and Inodon ${ }^{\circledR}$ showed great differences in $\mathrm{pH}$, respectively $(4,96)$ and $(4,65)$, ie above $30 \%$ between the expected and found $\mathrm{pH}$. The densities of the evaluated gels indicate this products with appropriate values for the purpose intended (topical application of fluoride overseen by Dentist), however the products Odahcam ${ }^{\circledast}$ and Onodon ${ }^{\circledast}$ showed great differences in $\mathrm{pH}$, placing them outside the minimum standard of effectiveness desired.

Key words: ACIDULATED PHOSPHATE FLUORIDE; HYDROGEN-ION CONCENTRATION; SODIUM FLUORIDE. 


\section{INTRODUÇÃO}

Atualmente, o emprego de fluoretos seja na forma de administração sistêmica (uso interno ou enteral), ou na forma de aplicação tópica (uso externo ou parenteral), é ato pragmático e quase que uma unanimidade em termos de Odontologia Preventiva. Sabe-se perfeitamente que o tratamento meramente curativo, restaurador ou reabilitador quando destituído de amparo preventivo adjuvante ou principal, é invariavelmente fadado ao insucesso, tanto do ponto de vista da eficiência clínica, quanto, inclusive, no que tange aos custos financeiros ${ }^{1}$.

Dentre as características dos géis fluoretados para aplicação tópica, a densidade e o pH destes produtos possuem importância muito significativa na segurança e eficácia deste procedimento preventivo. O propósito de um tratamento com aplicação de flúor $\left(\mathrm{F}^{-}\right)$por um profissional é beneficiar o esmalte dentário por meio de um efeito tópico, não pela ingestão sistêmica. Por essa razão, em um procedimento de aplicação tópica de fluoreto tido como "ideal", o paciente não deveria deglutir fluoreto algum. ${ }^{2,3}$

Entretanto muitas variáveis podem afetar a chamada "rede intraoral de retenção do fluoreto", e, por conseguinte, a ingestão do gel fluoretado, dentre elas o tipo de moldeira, a posição da cadeira no momento da aplicação, o tipo de gel empregado, a expectoração do paciente após a aplicação tópica, e a densidade do gel. ${ }^{3,4}$

A medida desse parâmetro tem relação com a dispensação do gel para a utilização e a conseqüente veiculação dos princípios ativos. Essa determinação pode introduzir uma definição de quantidade de uso em função da faixa etária do usuário, podendo transformar-se na "dose" a ser empregada associando-se à moldeira, que também obedece à condição de individualização para criança, jovem e adulto. ${ }^{5-7}$

Um fator importante para afirmação nesse item é realçado por Manly ${ }^{8}$ (1943), que aconselha, em função das diferentes densidades dos produtos, orientar o volume de material a ser depositado sobre a moldeira. Como nova comprovação, Horowitz e Heifetz ${ }^{9}$ (1975), Arends et al. ${ }^{10}$ (1992) e Newbrun ${ }^{11}$ (2001), confirmam o valor da verificação da densidade em suas pesquisas, à medida que afirmam ser a quantidade de dentifrício ou gel fluoretado colocada sobre as cerdas das escovas dentais o fator mais importante e que promove a ingestão maior ou menor de fluoreto pela criança.

Em relação ao $\mathrm{pH}$ dos géis fluoretados, podese afirmar que é uma das características mais importantes e responsável pela sua estabilidade química e atividade declarada. Os géis com $\mathrm{pH}$ ácido têm maior estabilidade no meio bucal $\mathrm{e}$, portanto, maior atividade antimicrobiana $\mathrm{e}$ remineralizadora. ${ }^{12}$ Caracterizados como produtos de ação prolongada, na qual se insere a retenção intraoral, os géis de fluoretos têm dependência do pH para que essa interação ocorra. ${ }^{13-15}$

Além disso, o pH provoca também variações na viscosidade final dos produtos de higiene oral. ${ }^{16}$ Lammers e Driessens ${ }^{15}$ (1992) chamam ainda a atenção para o processo de remineralização do esmalte dental, com o ajuste do pH para 6,8.

Cumpre salientar que, dentre todos os procedimentos para aplicação tópica de fluoretos em consultório dentário, a saber: soluções fluoretadas, géis fluoretados, vernizes fluoretados e pastas profiláticas fluoretadas, a técnica mais universalmente empregada é com o flúor fosfato acidulado (FFA), a $1,23 \%$ na forma de gel a 12.300 partes por milhão de flúor (ppmF-) dispensado em moldeiras. ${ }^{17-20}$

Com base nessa premissa, o objetivo do presente trabalho foi analisar a densidade e o pH de géis fluoretados disponíveis no mercado nacional, de diferentes marcas comerciais e em duas concentrações distintas.

\section{Material e métodos}

Para a verificação das propriedades dos géis fluoretados presentes no mercado brasileiro e, por conseguinte, avaliar a qualidade dos mesmos, foram adquiridos 10 produtos de cinco diferentes fabricantes, separados em géis de fluoreto de sódio a $2 \%$, com caráter neutro, e géis de FFA a $1,23 \%$, dados estes declarados pelos fabricantes e impressos nos rótulos das embalagens.

A tabela 1 contempla os produtos avaliados neste trabalho, bem como a concentração de fluoreto por unidade (forma de apresentação, ou seja, por frasco), o pH que os diferencia em géis acidulados e de caráter neutro, além dos fabricantes dos produtos. 
Tabela 1. Produtos avaliados, concentração por frasco, $\mathrm{pH}$ e fabricante.

\begin{tabular}{|c|c|c|}
\hline Produtos & [ ] & Fabricante \\
\hline 01. Inodon ${ }^{\circ}$ & 1,23\% - Acidulado & Inodon Laboratório Ltda. \\
\hline 02. Inodon ${ }^{\mathrm{O}}$ & $2,00 \%$ - Neutro & Inodon Laboratório Ltda. \\
\hline 03. Nupro ${ }^{\circ} T$ & $2,00 \%$ - Neutro & Dentsply Ind. Com. Ltda. \\
\hline 04. Odahcam ${ }^{\text {Ò }} T$ & 1,23\% - Acidulado & Dentsply Ind. Com. Ltda. \\
\hline 05. Flutop $\mathrm{Gel}^{\mathrm{O}} T$ & 1,23\% - Acidulado & SSWhite Art. Dents. Ltda. \\
\hline 06. Flutop $\mathrm{Gel}^{\mathrm{O}} T$ & $2,00 \%$ - Neutro & SSWhite Art. Dents. Ltda. \\
\hline 07. Topgel $^{\mathrm{O}} T$ & 1,23\% - Acidulado & Vigodent S.A. Ind. Com. \\
\hline 08. Topgel ${ }^{\mathrm{O}} T$ & $2,00 \%$ - Neutro & Vigodent S.A. Ind. Com. \\
\hline 09. Flugel $^{\mathrm{O}} T$ & $2,00 \%$ - Neutro & DFL Ind. Com. Ltda. \\
\hline 10. Sultan Topex ${ }^{\mathrm{O}} T$ & 1,23\% - Acidulado & DFL Ind. Com. Ltda. \\
\hline
\end{tabular}

Legenda: A letra "T" em itálico corresponde aos produtos declarados como tixotrópicos pelos fabricantes. O símbolo [ ] refere-se à concentração por frasco.

A tabela 2 apresenta os produtos quanto à data de fabricação, data de validade e número do lote adquirido. Importante salientar que algumas unidades estavam desprovidas de data de fabricação

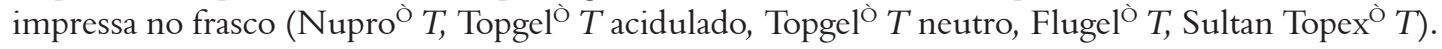

Tabela 2. Produtos avaliados, com data de fabricação, validade e lote.

\begin{tabular}{|c|c|c|c|}
\hline Produtos & Data Fabricação & $\begin{array}{c}\text { Data } \\
\text { Validade }\end{array}$ & $\begin{array}{c}\text { Lote } \\
\text { Número }\end{array}$ \\
\hline 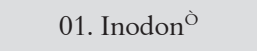 & $11 / 2002$ & $11 / 2004$ & 0011 \\
\hline 02. Inodon ${ }^{\mathrm{O}}$ & 09/2002 & 09/2004 & 0011 \\
\hline 03. Nupro ${ }^{\circ} T$ & ---------------------- & $11 / 2004$ & 68402 \\
\hline 04. Odahcam $^{\mathrm{O}} T$ & $10 / 2002$ & $10 / 2004$ & 7124 \\
\hline 05. Flutop $\mathrm{Gel}^{\mathrm{O}} T$ & $09 / 10 / 2002$ & 09/10/2004 & OOA \\
\hline 06. Flutop $\mathrm{Gel}^{\mathrm{O}} T$ & $26 / 08 / 2002$ & $26 / 08 / 2004$ & OPE \\
\hline 07. Topgel ${ }^{\mathrm{o}} T$ & -----------------' & $10 / 2005$ & 01402 \\
\hline 08. Topgel $^{\mathrm{O}} T$ & ------------------------ & 09/2005 & 01202 \\
\hline 09. Flugel $^{\mathrm{O}} T$ & ------------ & $05 / 2005$ & 0305369 \\
\hline 10. Sultan Topex ${ }^{\mathrm{O}} T$ & ------- & $04 / 2005$ & 0304351 \\
\hline
\end{tabular}

Legenda: Produtos em negrito, gel de fluoreto de sódio a $2 \%$. Produtos não negritados, gel de flúor fosfato acidulado a 1,23\%. Produtos acompanhados da letra "T" em itálico correspondem aos declarados como tixotrópicos pelos fabricantes. 
Apesar de todos os produtos que continham data de fabricação e data de expiração apresentarem dois anos de validade, o profissional que adquirir um dos produtos desprovidos de data de fabricação teria de ser informado da validade do produto em anos, para saber o tempo de estocagem no distribuidor, o que lhe possibilitaria maior segurança e confiabilidade na aplicação.

\section{DENSIDADE}

A avaliação da densidade das preparações foi realizada de acordo com metodologia proposta por Panzeri et al. ${ }^{21}$ (1979). Por meio de um dispositivo correspondente ao corpo de uma seringa hipodérmica cuja porção terminal foi removida, tornou-se possível medir 5 mililitros $(\mathrm{mL})$ de cada amostra, que foram submetidos à pesagem em balança analítica Mattler modelo H 10 W. Sobre o valor obtido nas pesagens foi aplicada uma fórmula para calcular o valor da densidade para cada produto testado (gel de fluoreto de sódio a $2 \%$, de caráter neutro e gel de flúor fosfato acidulado a 1,23\%, de caráter acidulado).

$p H$

Esse ensaio foi realizado segundo técnica preconizada por Panzeri et al. ${ }^{21}$ (1979), diluindose em um cálice de $15 \mathrm{~mL}$ a amostra do gel na proporção de 5 gramas (g) de gel (pesados em balança analítica Marte AS 2000) para $15 \mathrm{~mL}$ de água destilada (amostra: água destilada) sendo as leituras efetuadas em um pHmetro com eletrodo de vidro. Foi avaliado o pH dos 10 géis fluoretados para averiguar se o valor declarado pelos fabricantes correspondia à realidade.

\section{Resultados}

DENSIDADE

Os resultados obtidos da avaliação da densidade dos géis fluoretados com caráter neutro e com caráter ácido estão apresentados na Tabela 3.
Tabela 3. Resultados da Avaliação da Densidade dos géis fluoretados.

\begin{tabular}{|c|c|c|}
\hline & Produtos & Densidade \\
\hline & 01. Inodon ${ }^{\mathrm{O}}$ & 1,0834 \\
\hline & 02. Inodon ${ }^{\mathrm{O}}$ & 1,1086 \\
\hline & 03. Nuprò̀ $T$ & 1,1096 \\
\hline & 04. Odahcam ${ }^{\mathrm{ò}} T$ & 1,0942 \\
\hline \multirow{4}{*}{$T$} & 05. Flutop $\mathrm{Gel}^{\mathrm{O}} T$ & 1,1306 \\
\hline & 06. Flutop $\mathrm{Gel}^{\mathrm{O}}$ & 1,1238 \\
\hline & 07. Topgel ${ }^{\mathrm{O}} T$ & 1,0803 \\
\hline & 08. Topgel ${ }^{\grave{O}} T$ & 1,0633 \\
\hline \multirow{3}{*}{ Topex ${ }^{\mathrm{O}} T$} & 09. Flugel ${ }^{\mathrm{O}} T$ & 1,1530 \\
\hline & Sultan & 1,0840 \\
\hline & $\overline{\mathrm{X}}$ & 1,1030 \\
\hline
\end{tabular}

Legenda: Produtos em negrito, gel de fluoreto de sódio a $2 \%$. Produtos não negritados, gel de flúor fosfato acidulado (FFA) a 1,23\%. A letra "T" em itálico corresponde aos produtos declarados como tixotrópicos pelos fabricantes.

$p H$

Os resultados obtidos da avaliação dos valores de $\mathrm{pH}$ dos géis, tanto com caráter neutro quanto com caráter ácido, por meio de um pHmetro com eletrodo de vidro, estão apresentados na Tabela 4. 
Tabela 4. Resultados da avaliação do $\mathrm{pH}$ dos géis.

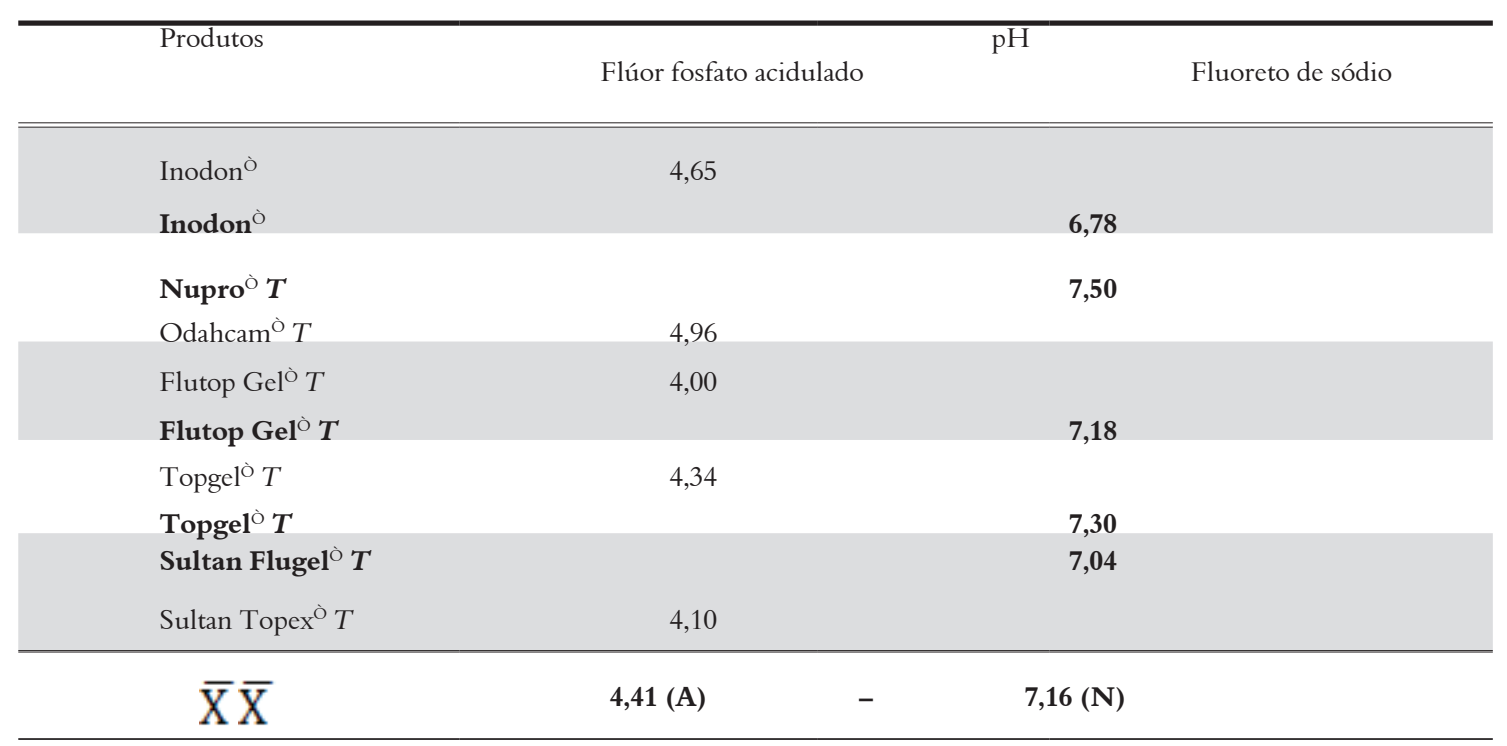

Legenda: A letra "T" em itálico corresponde aos produtos declarados como tixotrópicos pelos fabricantes.

\section{Discussão}

A medida da densidade é dependente das características dos componentes da formulação do produto, e ainda da existência ou não de ar incorporado durante o processo de mistura. Além disso, guarda relação com a dispensação do gel, e consequentemente com a veiculação do princípio ativo, no caso, fluoreto de sódio. ${ }^{1}$

Essa propriedade define a quantidade de uso em função da faixa etária do usuário, tornando-se a "dose" a ser empregada. Desta forma, poderia adequar-se a posologia para as faixas "criança", "jovem" e "adulto", condição essa que poderia minimizar ou até mesmo prevenir riscos de intoxicação aguda ${ }^{22,23}$.

Os valores de densidade dos géis fluoretados (média de 1,1030) das amostras selecionadas para este estudo apontam para produtos com valores adequados ao fim a que se destinam (aplicação tópica supervisionada por cirurgião-dentista).

O único senão fica por conta dos géis da Inodonò, que apresentaram incorporação excessiva de ar no produto final, talvez em função do emprego de carboximetilcelulose como agente espessante, já que, de acordo com Laba ${ }^{24}$ (1987), esse agente apresenta como característica peculiar o favorecimento de incorporação de bolhas de ar, se não adequadamente homogeneizado e em condições de vácuo. Essa incorporação de bolhas de ar deixou o produto com apresentação final pouco estética.
Fica clara a intenção de salvaguarda da saúde no exposto por Manly ${ }^{8}$ (1943), Hargreaves et al. ${ }^{25}$ (1972) e Naccache et al. ${ }^{26}$ (1992) que aconselham, em função das diferentes densidades dos produtos, orientar o volume de material a ser depositado sobre a moldeira. Também o emprego de moldeiras com revestimento interno "espumado" favorece a segurança de aplicação dos géis fluoretados, pois possibilita a retenção de maior massa crítica do gel. ${ }^{3}$

Deve-se ainda orientar o paciente para a expectoração do conteúdo bucal após a aplicação tópica de flúor (ATF), e o emprego de géis tixotrópicos, que representariam uma vantagem adicional na redução da retenção oral e, por conseguinte, da ingestão de fluoreto.

\section{Conclusónes}

As densidades dos géis avaliados apontam para produtos com valores adequados ao fim a que se destinam (ATF supervisionada por cirurgiãodentista). Os valores de $\mathrm{pH}$ para os géis de fluoreto de sódio (2\%) estão rigorosamente dentro dos padrões estabelecidos para esses produtos. Já os géis de flúor fosfato acidulado $(1,23 \%)$ Odahcam $^{\mathrm{o}}$ e Inodon ${ }^{\grave{O}}$ apresentaram grandes diferenças de $\mathrm{pH}$, respectivamente $(4,96)$ e $(4,65)$, ou seja, acima de $30 \%$ entre o $\mathrm{pH}$ esperado e o encontrado, o que coloca esses produtos fora do padrão mínimo de eficácia desejado. 


\section{REFERÊNCIAS BIBLIOGRÁFICAS}

1. PedrazziV.Avaliaçãodas propriedades físicas, químicas e da rotulagem de géis fluoretados disponíveis no mercado brasileiro, com vistas à eficácia e à segurança de uso [tese de livre docência] Ribeirão Preto: Faculdade de Odontologia de Ribeirão Preto da Universidade de São Paulo; 2005.

2. Wei SH, Connor CW Jr. Fluoride uptake and retention in vitro following topical fluoride applications. J Dent Res. 1983 Jul; 62(7):830-2.

3. LeCompte EJ, Rubenstein LK. Oral fluoride retention with thixotropic and APF gels and foam-lined and unlined trays. J Dent Res. 1984 Jan; 63(1):69-70.

4. Whitford GM. Fluoride in dental products: safety considerations. J Dent Res. 1987 May; 66(5):1056-60.

5. Lara EHG, Panzeri H. Estudo do comportamento reológico de algumas preparações básicas de dentifrícios na forma de pasta. Aerossol Cosméticos. 1992 Mai/ Jun; 14(78):36-45.

6. Pedrazzi V. A presença de sílica espessante e sílica abrasiva em formulações básicas de dentifrícios - alguns aspectos físicoquímicos e biológicos [dissertação de mestrado] Ribeirão Preto: Faculdade de Odontologia de Ribeirão Preto da Universidade de São Paulo; 1996.

7. Pedrazzi V, Lara EHG, Panzeri H. Sílica em Dentifrícios - aspectos físicos e físicoquímicos. Cosmetics \& Toiletries. 1999 Nov/Dez; 11:66-9.

8. Manly RSA. Structureless recurrent deposit on teeth. J Dent Res. 1943 Dec; 22(6):479-86.

9. Horowitz HS, Heifetz SB. Clinical tests of dentifrices. Pharmacol Ther Dent.1975; 2(3-4):235-44.

10. Arends J, Devries J, Ruben J. The effect of an experimental mouthrinse on enamel lesions remineralization in vitro. J Biol Buccale. 1992 Mar; 20(1):63-6.

11. Newbrun E. Topical fluorides in caries prevention and management: a North American perspective. J Dent Educ. 2001 Oct; 65(10):1078-83.

12. Plaizier-Vercammen JA, Lecluse E, Boute P, De Neve RE. Rheological properties of topical fluoride gels. Dent Mater. 1989 Sep; 5(5):301-5.

13. Rolla G, Loe H, Schiott CR. Retention of chlorhexidine in human oral cavity. Arch Oral Biol. 1971 Sep; 16(9):1109-16.
14. Gjermo P, Rolla G. The plaque-inhibiting effect of chlorhexidine-containing dentifrices. Scand J Dent Res. 1971; 79(2):126-32.

15. Lammers PC, Driessens FCM. Influence of fluoride and $\mathrm{pH}$ on "in vitro" remineralization of bovine enamel. Caries Res. 1992; 26(1):8-13.

16. Därr A. Tecnologia farmacêutica. Zaragoza: Editorial Acribia; 1981.

17. Ripa LW. An evaluation of the use of professional (operator-applied) topical fluorides. J Dent Res. 1990 Feb; 69(Special Issue): 786-96.

18. Newbrun E. Current regulations and recommendations concerning water fluoridation, fluoride supplements, and topical fluoride agents. J Dent Res. 1992 May; 71(5):1255-65.

19. van Rijkom HM, Truin GJ, van't Hof MA. A meta-analysis of clinical studies on the cariesinhibiting effect of fluoride gel treatment. Caries Res. 1998; 32(2):83-92.

20. Kriger L. Considerações gerais sobre a terapia tópica fluoretada - produtos de uso profissional (Géis Fluoretados). In: ABOPREV: Promoção de Saúde Bucal. São Paulo: Artes Médicas; 2003.

21. Panzeri H. Avaliação de dentifrícios. $1^{\mathrm{a}}$ parte: Consistência, densidade, $\mathrm{pH}$, "vida útil" e perda de água. Odontólogo Moderno. 1979 Fev; 6(2):4-12.

22. Draize JH, Woodarde G, Calvery HO. Methods for the study of irritation and toxicity of substances applied topically to the skin and mucous membranes. J Pharmacol Exp Ther. 1944 Dec; 82:377-90.

23. Pedrazzi V, Lara EHG, Panzeri H, Ogasawara MS. Análisis reológico de formulas básicas de dentífricos conteniendo sílice abrasiva y sílice espesante. Rev FOLA/ORAL. 1999 Mar/ Jun; 15:46-51.

24. Laba D. Rheological properties of cosmetics and toiletries. Cosmetics \& Toiletries. 1987; 12:63-8.

25. Hargreaves JA, Ingran GS, Wagg BJ. A gravimetric study of the ingestion of toothpaste by children. Caries Research. 1972; 6(3):237-43.

26. Naccache H, Simard PL, Trahan L, Brodeur JM, Demers M, Lachapelle D, Bernard PM. Factors affecting the Ingestion of dentifrice by children. Journal of Public Health Dentistry. 1992; 52(4):222-6.

Recebido: 23-7-2012 Aprovado: 5-11-2012 